0038-1098(94)00551-6

\title{
COLLECTIVE MODES IN A QUASI-ONE-DIMENSIONAL, TWO-COMPONENT ELECTRON LIQUID
}

\author{
B. Tanatar
}

Department of Physics, Bilkent University, Bilkent, 06533 Ankara, Turkey

\author{
(Received 13 June 1994 by G. Bastard)
}

\begin{abstract}
Under favorable conditions, a new collective mode besides the usual plasmons may exist in degenerate electron-hole liquids. We calculate the dispersion and damping of this new mode (called the acoustic plasmon mode) in a quasi-one-dimensional, two-component electron liquid. We carry out our calculations first within the random-phase approximation, then include the effects of local-field corrections using a Hubbard-like approximation. The latter decreases the acoustic plasmon dispersion.
\end{abstract}

Keywords:

\section{INTRODUCTION}

THE FUNDAMENTAL collective excitations of density fluctuations in degenerate plasmas are called plasmons. Their physical properties have been much studied in different dimensionalities under various approximations, and were instrumental in explaining a vast range of phenomena in solid state physics. Two-component plasmas (or more generally multicomponent plasmas) as in an electron-hole liquid can also support, other than the usual plasmon mode, a new collective mode called the "acoustic-plasmon mode" [1]. It has a long-wavelength dispersion $\omega_{q} \sim c q$ where $c$ is the velocity of propagation. In light scattering experiments Pinczuk et al. [2] have observed the acoustic mode in bulk GaAs samples. In lower dimensions no such observation has yet been reported. Dispersion and damping of the acoustic modes in bulk and two-dimensional electron-hole systems were studied theoretically within the randomphase-approximation (RPA) with [1] and without [3] the local-field corrections.

The main purpose of this communication is to investigate the dispersion and damping of acoustic plasmons in the quasi-one-dimensional (Q1D), twocomponent uniform electron liquid. It has recently been possible to fabricate artificial structures in which the charge carriers are confined to move essentially in one space dimension. The ordinary plasmon modes in Q1D systems have been predicted
[4] and observed [5, 6]. Their dispersion relation is of acoustic nature, but we reserve this latter term to a different collective mode. Here we study the acoustic plasmons in a Q1D, two-component system, and find in the long-wavelength limit the dispersion of these new modes behaves as $\omega_{q}^{2} \sim q^{2} \ln q$ similar to the ordinary plasmon dispersion relation [4]. Still we call these new excitations of the two-component plasma "acoustic plasmons" to distinguish them from the ordinary plasmons. The weak logarithmic $q$ dependence is a result of the effective Coulomb interaction between the charge carriers in a Q1D structure, and is essentially independent of the model describing them [7]. Acoustic plasmons in 1D systems such as a long DNA molecule have also attracted some attention [8]. We note that the collective mode in these systems is what we call ordinary plasmons here.

The specific model we use in our calculation for the Q1D, two-component electron fluid is developed by Das Sarma and Lai [9] and is applicable to the experimental realizations of semiconducting systems [6]. The charge carriers are assumed to be in a zero thickness $x y$-plane with a harmonic confinement potential in the $y$-direction so that the subband energies are $\epsilon_{n}=\Omega_{i}(n+1 / 2)$ where $\Omega_{i}(i$ stands for electrons or holes) describes the strength of the confining potential. In this work, we shall assume that both types of carriers are in their lowest subbands. 
Another motivation to study the acoustic plasmons in Q1D electron-hole systems comes from the fact that they may provide a pairing mechanism like the BCS theory which leads to a superconducting transition. Such an acoustic plasmon mediated superconductivity has been proposed [10] and elaborated [11] for 2D electronhole liquids. Possibility of superconductivity due to ordinary plasmons in quantum wires were also considered [12].

The rest of this paper is organized as follows. In the next section we outline the theoretical calculations leading to the dispersion and damping of ordinary and acoustic plasmons in Q1D, twocomponent systems. In Section 3 we present the results of our calculations and conclude with a brief summary.

\section{THEORY}

We consider Q1D electron fluid with two kinds of particles: light electrons with mass $m_{l}$ and heavy electrons (or holes) with mass $m_{h}$, and assume that each species has a parabolic single-particle dispersion $\epsilon_{l}(k)=k^{2} / 2 m_{i}(i=l$ or $h)$. The number densities of light and heavy particles are $n_{l}$ and $n_{h}$, respectively. The corresponding Fermi momenta and Fermi energies are $k_{F i}=\pi n_{i} / 2$, and $E_{F i}=k_{F i}^{2} / 2 m_{i}$. We introduce the following notation:

$$
\begin{gathered}
\delta \equiv \frac{k_{F l}}{k_{F h}}=\frac{n_{l}}{n_{h}}, \quad \rho \equiv \frac{m_{h}}{m_{l}}, \\
q_{t}=\frac{q}{k_{F i}}, \quad \text { and } \quad \omega_{i} \equiv \frac{\omega}{2 E_{F i}} .
\end{gathered}
$$

Here $q$ and $\omega$ are momentum and frequency variables. using three dimensionless quantities, the real and imaginary parts of the Lindhard polarizabilities $Q_{i}^{0}(q, \omega)=\left(e^{2} / \epsilon_{0}\right) F(q) \Pi_{i}^{0}(q, \omega)$ for different species are [4]

$\operatorname{Re} Q_{i}^{0}(q, \omega)=\frac{F(q)}{\pi a_{i} k_{F i} q_{i}} \ln \left|\frac{\omega_{i}^{2}-\omega_{i-1}^{2}}{\omega_{i}^{2}-\omega_{i+}^{2}}\right|$,

and

$\operatorname{Im} Q_{i}^{0}(q, \omega)=\left\{\begin{array}{cc}-F(q) /\left(a_{i} k_{F i} q_{i}\right) & \text { for } \omega_{i} \leqslant \omega \leqslant \omega_{t+}, \\ 0 & \text { otherwise }\end{array}\right.$

where $\omega_{i \pm}=\left|q_{i}^{2} / 2 \pm q_{i}\right|$, and $a_{i}=\epsilon_{0} / m_{i} e^{2}$ are the effective Bohr radii. The Coulomb interaction between the particles in our model Q1D system is given by [13] $\left(e^{2} / \epsilon_{0}\right) F(q)$ where $F(q)=\exp \left(b^{2} q^{2} / 4\right) K_{0}\left(b^{2} q^{2} / 4\right)$ in which $K_{0}(x)$ is the modified Bessel function, and $\epsilon_{0}$ is the background dielectric constant. The characteristic length $b=1 / \sqrt{m_{i} \Omega_{i}}$ is related to the confining potential strengths of heavy and light particles, and for simplicity we use throughout this paper the same value of $b$ for both species. For more realistic calculations this restriction may easily be relaxed.

The collective modes of a two-component degenerate plasma within the generalized RPA is obtained from the zeros of the equation [14]

$$
\begin{gathered}
\Delta(q, \omega)=\left[1-\psi_{l l}(q) Q_{l}^{0}(q, \omega)\right]\left[1-\psi_{h h}(q) Q_{h}^{0}(q, \omega)\right] \\
-\psi_{l h}^{2}(q) Q_{l}^{0}(q, \omega) Q_{h}^{0}(q, \omega)=0,
\end{gathered}
$$

in which $\psi_{i j}(q)=1-G_{i j}(q)$ for $i, j=l$ or $h . G_{i j}(q)$ are the static local-field corrections which describe the exchange-correlation effects between like and unlike particles. In this formulation the collective excitations are determined beyond the RPA. In the case when all $\psi_{i j}$ 's are equal to unity we recover the standard RPA expression. In the long-wavelength limit equation (3) yields the dispersion relation for ordinary plasmons

$$
\begin{aligned}
{\left[\Omega_{h}^{\mathrm{op}}\right]^{2}=} & \frac{1}{2}\left(\psi_{h h}+\psi_{l l} \delta_{\rho}\right) A+\left[\frac{1}{4}\left(\psi_{h h}+\psi_{l l} \delta \rho\right)^{2}\right. \\
& \left.-\left(\psi_{l l} \psi_{h h}-\psi_{l h}^{2}\right)^{2}\right]^{1 / 2} A
\end{aligned}
$$

where $A=\left(8 r_{s l} / \pi^{2}\right) F(q) q_{h}^{2} \delta \rho$. We have defined the dimensionless electron gas parameter for different species by $r_{s i}=\pi /\left(4 a_{i} k_{F i}\right)$. The ordinary plasmons exist in the region $\omega_{h}>\rho\left|q_{h}^{2} / 2+\delta q_{h}\right|$, above the upper boundary of the light-electron continuum. In the RPA, when we neglect the local-field corrections (i.e., $\psi_{i j}=1$ ) the ordinary plasmon dispersion simplifies to $\left[\Omega_{h}^{\mathrm{op}}\right]^{2}=A(1+\delta \rho)$.

For a two-component plasma with mass ratio $\rho \gg 1$, the equation $\operatorname{Re} \Delta(q, \omega)=0$ admits another solution (acoustic plasmon) for frequencies above all particle-hole excitations of the heavy particle, and below most particle-hole excitations of the light particle. To calculate the dispersion of acoustic plasmons, we take the static limit of the light-electron polarizability $Q_{l}^{0}(q)$ and obtain

$$
\left[\Omega_{h}^{\mathrm{ap}}\right]^{2}=\frac{w_{h+}^{2} \mathrm{e}^{D\left(q_{k}\right)}-\omega_{h-}^{2}}{\mathrm{e}^{D\left(q_{h}\right)}-1},
$$

where

$D\left(q_{h}\right)=\left(1-\psi_{l l} B\right) /\left[\psi_{h h} A^{\prime}-\left(\psi_{l l} \psi_{h h}-\psi_{l h}^{2}\right) A^{\prime} B\right]$,

in which $A^{\prime}=\left(4 r_{s l} / \pi^{2}\right) F(q) \delta \rho / q_{h}$, and

$B=\left(2 A^{\prime} / \rho\right) \ln \left|\frac{q_{h}^{2} / 2-\delta q_{h}}{q_{h}^{2} / 2+\delta q_{h}}\right|$. 
The above expression in the long-wavelength limit of RPA gives $\Omega_{h}^{\mathrm{ap}} \approx(4 / \pi)\left(r_{s l} \delta \rho\right)^{1 / 2} q_{h}$ where we have also neglected the weak logarithmic singularity.

The damping of the acoustic plasmons, namely the rate at which they decay into particle-hole pairs is calculated (in units of $2 E_{F h}$ ) by [15]

$\gamma_{q}=\frac{\operatorname{Im} \Delta\left(q, \Omega_{h}^{\mathrm{ap}}\right)}{[\partial \operatorname{Re} \Delta(q, \omega) / \partial \omega]_{\omega=\Omega_{h}^{\mathrm{ap}}}}$.

The above expression is valid to first order in $\gamma_{q} / \Omega_{h}^{\text {ap }}$.

\section{RESULTS AND DISCUSSION}

We illustrate the existence of an acoustic plasmon in a Q1D, two-component electron fluid in Fig. 1, by choosing a system with particle number ratio $\delta=1$, mass ratio $\rho=3$, and density parameter for light particles $r_{s l}=1$. We also take the characteristic length $b$ in the effective Coulomb interaction to be equal to the Bohr radius $a_{l}$ of the light particles. The horizontally and vertically hatched regions show the heavy and light-electron single-particle continua, respectively. The ordinary plasmon which lies above the light-electron single-particle excitation region boundary $\rho\left|q_{h}^{2} / 2+\delta q_{h}\right|$ is indicated by the dashed line. The acoustic plasmon dispersion is shown by the solid line and remains in the region $\left|q_{h}^{2} / 2+q_{h}\right|<$ $\Omega_{h}^{\mathrm{ap}}<\rho\left|q_{h}^{2}-\delta q_{h}\right|$, above the upper boundary of heavy-electron continuum and below the lower boundary of light-electron continuum. In general, at larger wave vectors the acoustic plasmons either meet the upper boundary of the single-particle continuum of the heavy electrons at some critical wave vector $q_{c}$, or enter the light-electron continuum where it

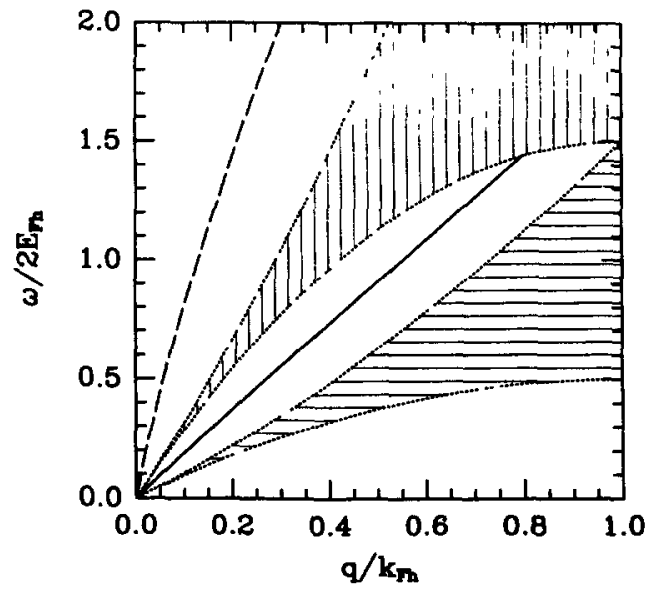

Fig. 1. The collective excitations and single particle domains for a Q1D, two-component electron liquid with parameters $\delta=1, \rho=3$, and $r_{s l}=1$. The solid and dashed lines indicate the ordinary acoustic plasmons, respectively. becomes heavily damped. We find for $\delta=1$ and $r_{s l}=1$ the acoustic plasmon branch will be present for all $\rho>1$. As the coupling strength is increased, the appearance of the acoustic plasmon is possibly only for some $\rho_{\min }$. For instance, at $\delta=1$, and $r_{s l}=3$ we find $\rho_{\min }=1.7$. Increase in $\delta$ has an effect of decreasing $\rho_{\min }$.

To include the effects of local-field corrections in our analysis, we take a simple Hubbard-like approximation [6] in which $G_{l h}(q)=0$, and

$G_{l, h h}(q) \approx \frac{1}{2} \frac{F\left(\sqrt{q_{l, h}^{2}+k_{F l, h}}\right)}{F\left(q_{l, h}\right)}$.

In Fig. 2, we display the changes brought about by the local-field corrections to the dispersion of ordinary and acoustic plasmons. Gold and Ghazali [7] have already noted the influence of Hubbard-like local-field corrections on the ordinary plasmons in a uniform Q1D electron system. We have taken $\delta=1$, $\rho=5$, and $r_{s l}=1$. The Hubbard approximation to the local-field corrections decrease the Q1D acoustic plasmon dispersion, a situation similar to the 2D case [3]. We note that the choice we make for the static $G_{i j}(q)$ may be improved considerably for a more realistic calculation by taking their selfconsistent values as in the case of $3 \mathrm{D}$ electron-hole liquids [14]. There are two main effects of the local-field corrections on the dispersion of acoustic phonons. First, as may be seen from Fig. 2 the wave vector $q_{c}$ at which the collective excitation enters the single-particle band increases. Second, $\rho_{\min }$

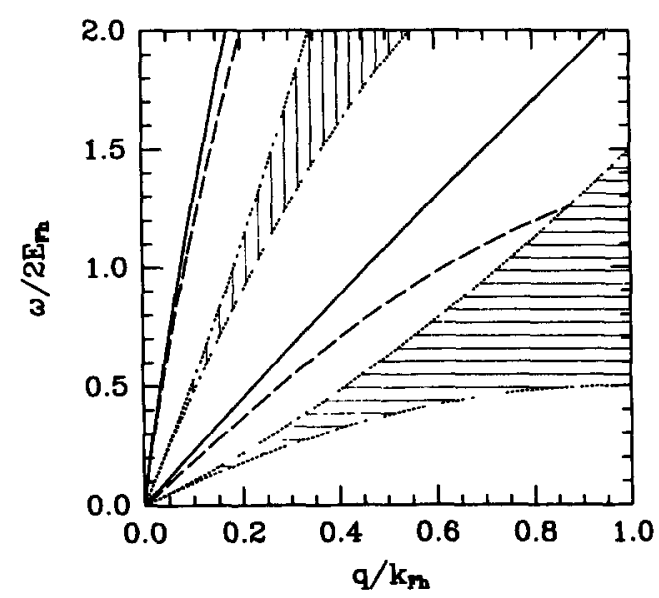

Fig. 2. The effect of local-field corrections within a Hubbard-like approximation on the collective excitations for a system with parameters $\delta=1$, $\rho=5$ and $r_{s l}=1$. Dashed and solid lines show the dispersion of ordinary and acoustic plasmons with and without the local-field corrections, respectively. 
slightly decreases upon the inclusion of $\psi_{i j}$ via a Hubbard-like approximation.

In contrast to the $3 \mathrm{D}$ and $2 \mathrm{D}$ cases, in $1 \mathrm{D}$ ordinary plasmons do not undergo Landau damping. Similarly, the acoustic plasmons when they appear do not exhibit this decay process into the particlehole pairs. Damping becomes effective, as the plasmons enter the heavy or light-electron continua, quantitatively described by the expression given in equation (6). To obtain damping for the collective excitations of a Q1D system, one needs to go beyond the static local-field corrections, i.e., to consider the frequency dependent $G_{i j}(q, \omega)$, which is beyond the scope of our consideration.

In summary, we have studied the acoustic plasmons in Q1D, two-component electron liquids. The dispersion and damping of these collective modes are calculated with and without the localfield corrections within the RPA. The effects of the local-field corrections (here calculated within the Hubbard approximation) seem to decrease the frequency and maximum wave vector for propagation of ordinary and acoustic plasmons, and increase the damping. Although we have used a particular model to describe the effective interactions among the Q1D charge carriers, and a crude form of the local-field corrections our results are expected to give quite generally the qualitative features of collective excitations in Q1D electron-hole systems. Experiments to observe the acoustic plasmons in Q1D structures, and their possible pairing mechanism leading to superconductivity would be most interesting.

Acknowledgements - This work is partially supported by the Scientific and Technical Research Council of Turkey (TUBITAK). We thank Dr E. Tekman for useful discussions.

\section{REFERENCES}

1. D. Pines, Can J. Phys. 34, 1379 (1956); J. Ruvalds, Adv. Phys. 30, 677 (1981).

2. A. Pinczuk, J. Shah \& P.A. Wolf, Phys. Rev. Lett. 47, 1487 (1981).

3. G. Vignale \& K.S. Singwi, Solid State Commun. 44, 259 (1982); G. Vignale, Phys. Rev. B38, 811 (1988); K.El Sayed, R. Binder, D-C. Scott \& S.W. Koch, Phys. Rev. B47, 10210 (1993); M.R.A. Shegelski \& D.J.W. Geldart, Phys. Rev. B40, 3647 (1989).

4. P.F. Williams \& A.N. Bloch, Phys. Rev. B10, 1097 (1974); W.I. Friesen \& B. Bergersen, J. Phys. C13, 6627 (1980).

5. A.R. Goñi, A. Pinczuk, J.S. Weiner, J.M. Calleja, B.S. Dennis, L.N. Pfeiffer \& K.W. West, Phys. Rev. Lett. 67, 3298 (1991).

6. See for instance, T. Demel, D. Heitmann, P. Grambow \& K. Ploog, Phys. Rev. B38, 12732 (1988).

7. For plasmons in a different model of Q1D systems, see for example, A. Gold \& A. Ghazali, Phys. Rev. B41, 7626 (1990).

8. L.L. Van Zandt \& V.K. Saxena, Phys. Rev. Lett. 61, 1788 (1988); J.B. Sokoloff, Phys. Rev. Lett. 63, 2316 (1989).

9. S. Das Sarma \& W.Y. Lai, Phys. Rev. B32, 1401 (1985).

10. J. Ruvalds, Phys. Rev. B35, 8869 (1987).

11. G.S. Canright \& G. Vignale, Phys. Rev. B39, 2740 (1989).

12. Y.C. Lee \& B.S. Mendoza, Phys. Rev. B39, 4776 (1989) (and references therein).

13. G.Y. Hu \& R.F. O'Connell, Phys. Rev. B42, 1290 (1990).

14. P. Vashishta, P. Bhattacharyya \& K.S. Singwi, Phys. Rev. B10, 5108 (1974).

15. A.L. Fetter \& J.D. Walecka, Quantum Theory of Many-Particle Systems. McGraw-Hill, New York (1971). 